\title{
The effect of interdialytic weight gain on pulmonary artery pressure and lung congestion in prevalent hemodialysis patients Magdy M. El Sharkawy ${ }^{1}$, Ahmed M. Samy EI Shimy ${ }^{2}$, Ali E. Abdel Rahman ${ }^{3}$, Ahmed M. Shebl Draz ${ }^{4}$, Lina Essam Khedr ${ }^{* 1}$ \\ Departments of ${ }^{1}$ Internal Medicine \& Nephrology and ${ }^{2}$ Radio Diagnosis, Faculty of Medicine, Ain Shams University, Egypt \\ Departments of ${ }^{3}$ Radiology and ${ }^{4}$ Nephrology, Nasser Institute Hospital, Egypt \\ *Corresponding author: Lina Essam Khedr, Mobile: (+20)1223421925, Email: linakhedr@med.asu.edu.eg
}

\begin{abstract}
Background: Patients with end stage renal disease on regular hemodialysis (HD) commonly present with volume overload. This has been found to be associated with increased incidence of pulmonary hypertension $(\mathrm{PH})$ and chronic lung congestion.

Objective: The aim of the study was to determine the effect of interdialytic weight gain (IDWG) on pulmonary artery pressure by echocardiography and lung congestion by lung ultrasound.

Patients and Methods: This observational cross-sectional study was conducted on 60 patients on regular haemodialysis at Nasser Institute Hospital, Cairo. Patients were divided into 2 groups: Group (I) 30 patients with IDWG < 3.5\% of their dry weight, and Group (II) 30 patients with IDWG $>3.5 \%$ of their dry weight.

Results: Patients with increased IDWG $>3.5 \%$ (group 2) had pre-HD higher pulmonary artery pressure (PAP) than those with IDWG $<3.5 \%$ (group 1) and patients with IDWG $>3.5 \%$ had more change in their PAP post HD session than those with IDWG $<3.5 \%$ but no significant difference between the change after the HD session in both groups. There is a significant relation between HD duration, anemia, and hypercalcemia with PH. Patients with more increased IDWG $>3.5 \%$ (group 2) had higher pulmonary congestion than those with IDWG < 3.5\% (group 1) and patients with IDWG $<3.5 \%$ had more change in their lung congestion level after the HD session than those with IDWG $>3.5 \%$ but no statistical significance between the change in both groups.
\end{abstract}

Conclusion: Increase in IDWG\% over 3.5\% significantly affects lung congestion and pulmonary hypertension in dialysis patients.

Keywords: Pulmonary Hypertension, pulmonary congestion, Haemodialysis, Interdialytic weight gain.

\section{INTRODUCTION}

Pulmonary hypertension $(\mathrm{PH})$ is a recognized cardiovascular complication for patients with end stage renal disease (ESRD) on hemodialysis (1). Echocardiography is a non-invasive, vastly available and inexpensive tool for screening for $\mathrm{PH}$ and monitoring its progression ${ }^{(2)}$.

The prevalence of pulmonary hypertension in hemodialysis patients is high and differs in different studies from $17 \%$ to $49.53 \%$ depending on the method of dialysis and the presence of other cardiovascular comorbidities ${ }^{(3)}$.

Lung congestion is a common problem in ESRD patients on dialysis. Before hemodialysis (HD), nearly $60 \%$ of ESRD patients show moderate to severe lung congestion which was decreased but not completely abolished by ultrafiltration process during dialysis, and nearly one third to one fourth of those patients had some degree of lung congestion post hemodialysis ${ }^{(4)}$. Marini reported in 2017 that lung ultrasound can be used as a reliable method of evaluation of lung congestion through assessment of B-lines ${ }^{(5)}$. A B-line score was calculated and categorized as minimal (0-5 B-lines present), mild (6-13), moderate (14-30) and severe (>30 B) pulmonary congestion ${ }^{(\boldsymbol{(})}$.

The aim of the current work was to determine the effect of interdialytic weight gain (IDWG) on pulmonary hypertension using echocardiography (Echo) and lung congestion using lung ultrasound (LUS).

\section{PATIENTS AND METHODS}

This comparative cross-sectional study included a total of 60 patients on regular haemodialysis, at Nasser Institute Hospital, Cairo.

Patients were divided into 2 groups: Group (I) 30 patients with IDWG $<3.5 \%$ of their dry weight, and Group (II) 30 patients with IDWG $>3.5 \%$ of their dry weight. Comparison between the two groups was done to detect statistically the effect of IDWG and ultrafiltration on PAP and lung congestion.

A standardized questionnaire used to collect demographic data and data regarding the kidney disease and HD including aetiology of ESRD, date of onset of haemodialysis therapy and HD access. Laboratory data including haemoglobin, calcium, phosphorus, urea and virology were also collected.

Pulmonary artery pressure was measured by echo and patients classified according to PAP into mild (25$30 \mathrm{mmHg}$ ) moderate $(30-44 \mathrm{mmHg})$ and severe $(>45$ $\mathrm{mmHg}$ ) pulmonary hypertension. Lung congestion in all patients was measured by lung ultrasound and patients classified into minimal (0-5 B lines) mild (613) moderate (14-30) and severe (>30) lung congestion. ESRD patients' age $\geq 18$ years old had been on regular haemodialysis 3 times per week $>6$ months at Nasser 
Institute hospital in the province of Cairo. we excluded patients with Primary pulmonary Hypertension, Severe anaemia (HB < 9), Heart failure "EF < $45 \%$ ", Pulmonary Hypertension secondary to lung fibrosis, sarcoidosis, IPF and COPD, AVF flow rate > 1.5 litre/min, Mitral valve and Aortic valve diseases, uncontrolled Hypertension, previous venous thromboembolism.

\section{Haemodialysis method:}

HD patients receiving sessions with blood flow rate and the dialysate flow rate were $200-400 \mathrm{~mL} / \mathrm{min}$ and $500 \mathrm{~mL} / \mathrm{min}$ respectively. The patients received treatment with synthetic dialyzer membranes (polysulfone) and bicarbonate-based dialysis solution with $35-40 \mathrm{mEq} / \mathrm{L}$ bicarbonate concentration.

Echocardiograms: Echocardiogram with twodimensional (2D) and guided M-mode. PH was defined as a mean PAP equal to or higher than $25 \mathrm{~mm} \mathrm{Hg}$.

Lung ultrasound: Two-dimensional (2D) and guided M-mode ultrasound were performed by radiologist with a digital ultrasound machine as a non-invasive method. Lung congestion is classified as minimal (0-5 B lines), mild (6-13 B lines), moderate (14-30 B lines) and severe (>30 B lines) lung congestion.

Laboratory assay: Blood sampling for assay of haemoglobin, calcium, phosphorus urea and virology was taking and assessed immediately before the dialysis session by HD staff and the serum was separated immediately.

\section{Ethical consent:}

An approval of the study was obtained from Ain Shams University Academic and Ethical Committee number FWA000017585. Every patient signed an informed written consent for acceptance of the operation. This work has been carried out in accordance with The Code of Ethics of the World Medical Association (Declaration of Helsinki) for studies involving humans.

\section{Statistical analysis:}

Recorded data were analyzed using the statistical package for social sciences, version 20.0 (SPSS Inc., Chicago, Illinois, USA). Quantitative data were expressed as mean \pm standard deviation (SD). Qualitative data were expressed as frequency and percentage. The following tests were done: Independent-samples t-test of significance was used when comparing between two means. Paired sample ttest of significance was used when comparing between related samples. Chi-square $\left(\mathrm{x}^{2}\right)$ test of significance was used in order to compare proportions between qualitative parameters. Pearson's correlation coefficient (r) test was used to assess the degree of association between two sets of variables. The confidence interval was set to $95 \%$ and the margin of error accepted was set to $5 \%$. So, the p-value was considered significant as the following: Probability (P-value). P-value $<0.05$ was considered significant. P-value $<0.001$ was considered as highly significant. P-value $>0.05$ was considered insignificant.

\section{RESULTS}

Table (1) shows statistically significant relation between pulmonary hypertension with longer hemodialysis duration ( $p<0.001)$, IDWG\% ( $p=0.011$ ) hemoglobin level $(p=0.021)$ and calcium levels $(\mathrm{p}<0.001)$. The mean of pre HD pulmonary artery pressure was $21.83 \pm 7.55$, post HD level was $18.18 \pm 6.31$, with change $\%-16.17 \%$, no patient showed severe $\mathrm{PH}$ and prevalence of pulmonary hypertension in the study group is $30 \%$ and decreased after dialysis to 21.7\%.In group (I) 24 patients had PAP $<25 \mathrm{mmHg}$ and 6 had mild Degree of PH, in group (II), 18 patients had no $\mathrm{PH}, 9$ had mild and 3 had moderate.

Table (2) shows statistically significant difference between group I and group II regarding to pre HDx, Post HDx of pulmonary hypertension. Patients with increased IDWG $>3.5 \%$ had pre HD pulmonary artery pressure $(24.33 \pm 7.50 \mathrm{mmHg})$, higher than those with IDWG $<3.5 \%(19.33 \pm 6.83 \mathrm{mmHg})$ (p value $0.009)$. Furthermore, patients with IDWG $>3.5 \%$ had more change in their pulmonary artery pressure the HD session than those with IDWG $<3.5 \%$. Table (3) shows statistically significant change between pre and post pulmonary Hypertension in each group that prove that HD therapy is effective in lowering PH severity.

Table (4) shows statistically significant relation between lung congestion with hemodialysis duration $(p=0.005)$, IDWG\% $(p<0.001)$ and hemoglobin level $(\mathrm{p}=0.035)$.

The mean pre-HD B lines by lung ultrasound were $14.45 \pm 3.40$, post HD level 5.20 \pm 2.33 , with change $\%-63.79 \%$ and that all patients have lung congestion that improved by HD session but not completely abolished. In group (I) 17 patients had mild pre dialysis lung congestion and 13 had moderate, while in group (II), only 7 patients had mild lung congestion and 23 had moderate lung congestion which was statistically significant with $\mathrm{p}$ value (0.008).

Similarly, post dialysis in only 7 patients had mild congestion while 23 had minimal lung congestion in group (I). In group (II) 17 patients had mild and 13 patients had minimal lung congestion post dialysis ( $\mathrm{p}$ value 0.008 ).

Multi-Regression analysis was done for pulmonary HTN and variables that showed statistically significant. It showed that Hemodialysis duration (years), IDWG\% and Calcium have significance while the $\mathrm{Hb}$ was insignificant.

Multi-Regression analysis was done for lung congestion and variables that showed statistically significant. It showed that Hemodialysis duration (years), IDWG\% and hemoglobin have significance while calcium was insignificant. 
Table (1): Relation between pulmonary hypertension according to sex, age (years), vascular access, hemodialysis duration (years), IDWG\%, anemia, calcium and $\mathrm{PO} 4$ in all patients.

\begin{tabular}{|c|c|c|c|c|c|}
\hline \multirow{2}{*}{ Parameters } & \multicolumn{3}{|c|}{ Pulmonary Hypertension } & \multirow{2}{*}{$\mathbf{t} / \mathbf{x} 2 \#$} & \multirow{2}{*}{ p-value } \\
\hline & No & Mild & Moderate & & \\
\hline \multicolumn{6}{|l|}{ Sex } \\
\hline Female & $14(33.3 \%)$ & $7(46.7 \%)$ & $0(0.0 \%)$ & \multirow{2}{*}{$2.564 \#$} & \multirow{2}{*}{0.277} \\
\hline Male & $28(66.7 \%)$ & $8(53.3 \%)$ & $3(100.0 \%)$ & & \\
\hline Age (years) & $53.57 \pm 15.67$ & $51.13 \pm 13.09$ & $64.00 \pm 0.00$ & 0.949 & 0.393 \\
\hline \multicolumn{6}{|l|}{ Vascular access } \\
\hline Arteriovenous fistula & $38(90.5 \%)$ & $10(66.7 \%)$ & $3(100.0 \%)$ & \multirow{3}{*}{ 5.592\# } & \multirow{3}{*}{0.232} \\
\hline $\begin{array}{l}\text { Temporary double lumen } \\
\text { intravascular catheter }\end{array}$ & $2(4.8 \%)$ & $3(20.0 \%)$ & $0(0.0 \%)$ & & \\
\hline $\begin{array}{l}\text { Permanent double lumen } \\
\text { intravascular catheter }\end{array}$ & $2(4.8 \%)$ & $2(13.3 \%)$ & $0(0.0 \%)$ & & \\
\hline Hemodialysis duration (years) & $6.23 \pm 2.60$ & $13.27 \pm 6.34$ & $11.33 \pm 1.15$ & 19.526 & $<0.001 * *$ \\
\hline IDWG\% & $3.41 \pm 1.54$ & $4.41 \pm 1.90$ & $5.90 \pm 0.00$ & 4.860 & $0.011 *$ \\
\hline Hemoglobin (g/dl) & $10.44 \pm 1.42$ & $9.34 \pm 0.84$ & $10.30 \pm 0.01$ & 4.139 & $0.021 *$ \\
\hline Calcium (mg/dl) & $8.29 \pm 0.66$ & $8.99 \pm 0.62$ & $9.00 \pm 0.01$ & 7.691 & $<0.001 * *$ \\
\hline PO4 (mg/dl) & $4.58 \pm 1.06$ & $3.95 \pm 0.19$ & $4.30 \pm 0.01$ & 1.857 & 0.165 \\
\hline
\end{tabular}

Table (2): Comparison between pre-HD and post HD pulmonary Hypertension in group I and group II.

\begin{tabular}{|l|c|c|c|c||}
\hline Pulmonary Hypertension & Group I (n=30) & Group II (n=30) & t-test & Sig. \\
\hline \hline $\begin{array}{l}\text { Pre HD level } \\
\text { Mean } \pm \text { SD }\end{array}$ & $19.33 \pm 4.83$ & $24.33 \pm 3.50$ & 7.281 & $0.009^{*}$ \\
\hline $\begin{array}{l}\text { Post HD level } \\
\text { Mean } \pm \text { SD }\end{array}$ & $16.18 \pm 3.70$ & $20.17 \pm 4.12$ & 6.535 & $0.013^{*}$ \\
\hline $\begin{array}{l}\text { Difference } \\
\text { Mean } \pm \text { SD }\end{array}$ & $-14.72 \pm 3.46$ & $-17.61 \pm 3.48$ & 1.731 & 0.193 \\
\hline
\end{tabular}

t-Independent Sample t-test;

p-value $>0.05 \mathrm{NS}$; ${ }^{*}$-value $<0.05 \mathrm{~S}$

Table (3): Comparison between pre-HD and post HD changes in pulmonary Hypertension in each group.

\begin{tabular}{|l|c|c|}
\hline Pulmonary HTN & Group I (n=30) & Group II (n=30) \\
\hline \hline Pre-HD & $19.33 \pm 6.83$ & $24.33 \pm 7.50$ \\
\hline Post HD & $16.18 \pm 4.70$ & $20.17 \pm 7.12$ \\
\hline Difference & $-14.72 \pm 9.46$ & $-17.61 \pm 7.48$ \\
\hline Paired sample t-test & 6.306 & 15.809 \\
\hline p-value & $0.021^{*}$ & $0.014^{*}$ \\
\hline
\end{tabular}

Table shows statistically significant change between pre and post pulmonary Hypertension in each group that prove that HD therapy is effective in lowering PH severity. 
Table (4): Relation between lung congestion according to sex, age (years), vascular access, hemodialysis duration (years), IDWG\%, anemia, calcium and PO4 in all patients.

\begin{tabular}{||l|c|c|c|c||}
\hline \multirow{2}{*}{ Parameters } & \multicolumn{2}{|c|}{ Lung congestion } & \multirow{2}{*}{ t/x2\# } & \multirow{2}{*}{ p-value } \\
\cline { 2 - 3 } & Mild & Moderate & & \\
\hline \hline Sex & & & & \\
\hline Female & $7(29.2 \%)$ & $14(38.9 \%)$ & \multirow{2}{*}{$0.598 \#$} & \multirow{2}{*}{0.439} \\
\hline Male & $17(70.8 \%)$ & $22(61.1 \%)$ & & \\
\hline Age (years) & $53.75 \pm 11.85$ & $53.31 \pm 16.60$ & 0.013 & 0.910 \\
\hline Vascular access & & & \\
\hline Arteriovenous fistula & $17(70.8 \%)$ & $34(94.4 \%)$ & \multirow{2}{*}{$4.611 \#$} & \multirow{2}{*}{0.053} \\
\hline Temporary double lumen intravascular catheter & $5(20.8 \%)$ & $0(0.0 \%)$ & \\
\hline Permanent double lumen intravascular catheter & $2(8.3 \%)$ & $2(5.6 \%)$ & & \\
\hline Hemodialysis duration (years) & $10.38 \pm 6.27$ & $6.82 \pm 3.09$ & 8.517 & $0.005^{*}$ \\
\hline IDWG\% & $2.77 \pm 1.21$ & $4.46 \pm 1.67$ & 18.238 & $<0.001^{* *}$ \\
\hline Anemia & $9.75 \pm 1.33$ & $10.43 \pm 1.29$ & 3.893 & $0.035^{*}$ \\
\hline Calcium & $8.48 \pm 0.80$ & $8.51 \pm 0.64$ & 0.014 & 0.906 \\
\hline PO4 & $4.43 \pm 1.07$ & $4.39 \pm 1.12$ & 0.011 & 0.917 \\
\hline \hline
\end{tabular}

Table (4) shows statistically significant relation between lung congestion with hemodialysis duration $(\mathrm{p}=0.005)$, IDWG\% $(\mathrm{p}<0.001)$ and hemoglobin level $(\mathrm{p}=0.035)$.

Table (5): Comparison between pre and post HD changes in lung congestion in each group.

\begin{tabular}{|l|c|c||}
\hline \multicolumn{1}{|c|}{ Lung congestion } & Group I $(\mathbf{n}=\mathbf{3 0})$ & Group II $(\mathbf{n}=\mathbf{3 0})$ \\
\hline \hline Pre HDx & $12.83 \pm 2.31$ & $16.07 \pm 3.58$ \\
\hline Post HDx & $4.33 \pm 2.25$ & $6.07 \pm 2.12$ \\
\hline Change\% & $-65.51 \pm 17.20$ & $-62.07 \pm 10.42$ \\
\hline Paired sample t-test & 12.836 & 20.551 \\
\hline p-value & $<0.001^{* *}$ & $<0.001^{* *}$ \\
\hline
\end{tabular}

Table (6): Correlation between volume removed with pre HD pulmonary hypertension and pre HD lung congestion in the all patients.

\begin{tabular}{|l|c|c|}
\hline \multirow{2}{*}{ All patients (n=60) } & \multicolumn{2}{c|}{ Volume removed (liter) } \\
\cline { 2 - 3 } & $\mathbf{R}$ & p-value \\
\hline Pulmonary HTN: Pre HDx & 0.098 & 0.459 \\
\hline Lung congestion: Pre HDx & 0.626 & $<0.001 * *$ \\
\hline
\end{tabular}

r-Pearson Correlation

p-value >0.05 NS; *p-value <0.05 S; **p-value <0.001 HS

Table (7): Multivariate regression analysis of pulmonary HTN.

\begin{tabular}{|l|c|c|c|}
\hline \multicolumn{1}{|c|}{ Parameters } & Standardized regression $(\square)$ & T & Sig. \\
\hline Hemodialysis duration (years) & 0.566 & 6.824 & $<0.001^{* *}$ \\
\hline IDWG\% & 0.413 & 5.729 & $<0.001^{* *}$ \\
\hline Hb. & -0.109 & -1.431 & 0.158 \\
\hline Calcium & 0.232 & 2.791 & $0.007^{*}$ \\
\hline
\end{tabular}

Table (8): Multivariate regression analysis of lung congestion.

\begin{tabular}{|l|c|c|c|}
\hline \multicolumn{1}{|c|}{ Parameters } & Standardized regression $(\square)$ & T & Sig. \\
\hline Hemodialysis duration (years) & -0.226 & -2.449 & $0.018^{*}$ \\
\hline IDWG\% & 0.670 & 8.345 & $<0.001^{* *}$ \\
\hline $\mathrm{Hb}$. & 0.237 & 2.810 & $0.007^{*}$ \\
\hline Calcium & -0.052 & -0.565 & 0.574 \\
\hline
\end{tabular}




\section{DISCUSSION}

Pulmonary hypertension $(\mathrm{PH})$ no matter its etiology, is a strong independent predictor of cardiovascular mortality in hemodialysis (HD) patients (7). In a review by Yigla et al. ${ }^{(8)}$, the prevalence of PH in ESRD patients was around 40\%-50\%. Prevalence of pulmonary hypertension is usually higher in hemodialysis patients especially those with arteriovenous fistula (AVF) ${ }^{(9)}$. In the current study, 60 patients were included, 18 had some degree of pulmonary hypertension, of those 12 had inter-dialytic weight gain over $3.5 \%$ of their dry weight. Although pulmonary artery pressure improved after ultrafiltration, 13 patients remained to have PAP $>25 \mathrm{mmHg}$.

There are many risk factors for $\mathrm{PH}$ in dialysis patients among them, age (10), hyperparathyroidism, possibly through pulmonary artery calcifications (11), uremic toxins, endothelial dysfunction, chronic inflammation, malnutrition, alongside mineral bone disorders, all have been implicated in the pathophysiology of pulmonary hypertension in dialysis patients ${ }^{(12)}$.

In our study calcium levels were found to be associated with higher pulmonary artery pressure supporting the previous findings. Furthermore, the duration of HD therapy was positively correlated with elevated PAP measured prior to the session. This finding may be related to intermittent chronic hypoxia and chronic uremia over years. A study done by Faqih et al. ${ }^{(13)}$, also observed the same association between hemodialysis duration and $\mathrm{PH}$. In a multi regression analysis for risk factors for $\mathrm{PH}$ in this study, dialysis duration, calcium and IDWG were all found to be statistically significant factors contributing to $\mathrm{PH}$.

We observed that hemoglobin level was lower in patients with higher pulmonary artery pressure. Hyper dynamic circulation secondary to anemia or increased flow in arteriovenous access may contribute to elevated pulmonary artery pressure ${ }^{(\mathbf{1 4})}$. However, we have excluded patients with AVF flows above 1.5 L/min, which could explain why patients with AVF did not differ as regards to pulmonary artery pressure than those patients with temporary catheters. Furthermore, hemoglobin level was not found statistically significant in a multiregression analysis of risk factors for $\mathrm{PH}$.

This study revealed that the mean change between pre-HD \& post HD PAP was $16 \%$ in all study groups. The mean difference between pre-and post HD session PAP in group (I) was (-14.72 \pm 9.46$)$ and the mean difference between pre-and post haemodialysis session PAP in group (II) was (-17.6 \pm 7.48$)$. Moreover, patients with increased IDWG $>3.5 \%$ had PAP pre-HD significantly higher than those with IDWG $<3.5 \%$ and patients with IDWG $>3.5 \%$ had more change in their PAP post HD session than those with IDWG $<3.5 \%$. These findings suggest that there may be an additive role for volume overload and increased interdialytic weight gain in between dialysis sessions in exacerbating pulmonary hypertension and elevated pulmonary blood flow that can't be accommodated by pulmonary vasculature. However, our study showed no statistically significant difference between group I and group II regarding the severity of pulmonary hypertension.

Lung ultrasound allows reliable clinical estimates of lung water. A study done by Zoccali $\boldsymbol{e t}$ al. (4) showed that lung ultrasound is an objective monitor of lung congestion in clinical practice. Two studies tested the prognostic value of lung US in patients admitted to a heart and lung department and patients with coronary heart disease ${ }^{(14-15)}$. In both studies, lung US predicted relevant clinical outcomes, including death and incident cardiovascular events.

In the current study, all patients had lung congestion of different degrees, $60 \%$ of them had moderate lung congestion while $40 \%$ had mild lung congestion and all of them had highly significant improvement of lung congestion by ultrafiltration.

However, 30 patients remained to have minimal and mild degrees of lung congestion post dialysis. Furthermore, a statistically significant difference between pre and post hemodialysis level of lung congestion between group I and group II was observed. Group II (with more interdialytic weight gain) observed more B lines in their lung ultrasound than group I (with less interdialytic weight gain). It has been suggested that excessive volume removal during dialysis can in itself contribute to increased weight gain in the interdialytic period ${ }^{(16)}$.

Pulmonary hypertension pathophysiology includes acute and chronic processes and HD therapy alone is not effective in completely improving pulmonary hypertension that may require additional medical treatment. Patients with increased IDWG > $3.5 \%$ (group 2) had pre-HD PAP significantly higher than those with IDWG $<3.5 \%$ (group 1). There was also a significant relation between anemia and hypercalcemia with $\mathrm{PH}$. Furthermore, there was a significant relation between IDWG with pulmonary congestion measured prior to the session when comparing to other significant parameters such as hemodialysis duration and hemoglobin levels.

\section{CONCLUSION}

It could be concluded that increase in IDWG\% over $3.5 \%$ significantly affects lung congestion and pulmonary hypertension in dialysis patients.

\section{ACKNOWLEDGMENTS}

Dr. MS conceived the idea, Dr.AD the data collection and analysis, Dr.LK wrote the manuscript. Dr AS and Dr AA performed the radiological procedures

\section{Disclosure of interest:}

All authors declare no conflict of interest. 


\section{Funding:}

No funding was applicable for this research.

\section{REFERENCES}

1. Meghan S, Courtwright A, Richard N (2013): Pulmonary hypertension in patients with chronic and end-stage kidney disease. Kidney International, 84: 682692.

2. Janda S, Shahidi N, Swiston J (2011): Diagnostic accuracy of echocardiography for pulmonary hypertension: a systematic review and meta-analysis. Heart, 97:61-66.

3. George G, Aguilera D, Carceles O et al. (2013): Pulmonary hypertension in dialysis patients. Renal Failure, 35: 514-520.

4. Zoccali C, Torino C, Tripepi R et al. (2013): Lung US in CKD Working Group: Pulmonary congestion predicts cardiac events and mortality in ESRD. J Am Soc Nephrol., 24: 639-646.

5. Marini C (2017): Lung ultrasound predicts decompensation in heart failure outpatients: another piece to the puzzle but still an incomplete picture. International Journal of Cardiology, 240: 324-325.

6. Saad M, Mansour W, Kamal J et al. (2015): B-Lines on lung ultrasound in end stage renal disease patients post hemodialysis: accuracy and precision-interim analysis. Annals of Emergency Medicine, 66: 4.

7. Yigla M, Fruchter O, Aharonson D et al. (2009): Pulmonary hypertension is an independent predictor of mortality in hemodialysis patients. Kidney International, 75(9): 969-975.

8. Yigla M, Abassi Z, Reisner S et al. (2009): Pulmonary hypertension in hemodialysis patients: an unrecognized threat. Seminars in Dialysis, 19: 353-357.
9. Nakhoul F, Yigla M, Gilman R et al. (2005): The pathogenesis of pulmonary hypertension in haemodialysis patients via arterio-venous access. Nephrology Dialysis Transplantation, 20: 16861692.

10. Harp R, Stavropoulos S, Wasserstein A et al. (2005): Pulmonary hypertension among end-stage renal failure patients following hemodialysis access thrombectomy. CardioVascular and Interventional Radiology, 28: 17-22.

11. Akmal M, Barndt R, Ansari A et al. (1995): Excess PTH in CRF induces pulmonary calcification, pulmonary hypertension and right ventricular hypertrophy. Kidney International, 47: 158-163.

12. Pabst S, Hammerstingl $C$, Hundt $F$ et al. (2012): Pulmonary hypertension in patients with chronic kidney disease on dialysis and without dialysis: results of the PEPPER-study. PLoS One, 7: 353-357.

13. Faqih $\mathrm{S}$, Noto-Kadou-Kaza $\mathrm{B}$, Abouamrane $\mathrm{L}$ et al. (2016): Pulmonary hypertension: prevalence and risk factors. Int J Cardiol Heart Vasc., 11:87-89.

14. Frassi F, Gargani L, Tesorio P et al. (2007): Prognostic value of extravascular lung water assessed with ultrasound lung comets by chest sonography in patients with dyspnea and/or chest pain. J Card Fail., 13:830-835.

15. Bedetti G, Gargani L, Sicari $R$ et al. (2010): Comparison of prognostic value of echographic (corrected) risk score with the Thrombolysis in Myocardial Infarction (TIMI) and Global Registry in Acute Coronary Events (GRACE) risk scores in acute coronary syndrome. Am J Cardiol., 106:1709-1716.

16. Hecking $M$, Karaboyas A, Antlanger $M$ et al. (2013): Significance of interdialytic weight gain versus chronic volume overload: consensus opinion. Am J Nephrol., 38: 78-90. 